\title{
Comparative Economic Studies Referees
}

\author{
Paul Wachtel ${ }^{1}$ \\ Published online: 16 April 2019 \\ (c) Association for Comparative Economic Studies 2019
}

This issue of Comparative Economic Studies includes the last articles that were submitted and evaluated while we were the editors. As we take leave of our 10-year guardianship of the journal, we would like to wish all the best to our successor, Nauro Campos. We would also like to express our gratitude to the referees who conscientiously assisted us in the evaluation of submitted manuscripts. Without their dedication, we would not have been able to maintain the high quality of the journal. Thus, it is our pleasure to take this opportunity to express our thanks to 302 individuals from all the over the globe who provided at least one (and often several) reviews since January 2016 when we last published such a list.

Josef Brada and Paul Wachtel

Sunday Adesina
Markus Ahlborn
Randall Akee
Alexandr Akimov
Michael Alexeev
Bernardina Algieri
Jamal Ali Alkhasawneh
Ageliki Anagnostou
Torben Andersen
John E. Anderson
Alin Andries
Muhammad Asali
Zeljka Asanovic
Panayiotis Athanasoglu
Jan Babecky
El-hadj Bah
Bas Berend Bakker

Paul Wachtel

Pwachtel@stern.nyu.edu

1 Stern School of Business, New York University, 44 West 4th Street, New York, NY 10012-1126, USA

Martin Brownbridge
Sam Bucovetsky
Christophe Cahn
Nauro Campos
Rosaria Rita Canale
Guglielmo Maria Caporale
Jeronimo Carballo
Vittorio Carlei
Richard L Carson
Oscar Carvallo
Nevin Cavusoglu
Stephen Cecchetti
Enzo Cerletti
Lucian Cernat
Geraldo Cerqueiro
Nildag Ceylan
Sheng-Tung Chen

Biswajit Banerjee
Thierno Barry
Will Bartlett
Furrukh Bashir
Yilmaz Bayar
Joscha Beckmann
Abdelaziz Benkhalifa
Hakan Berument
Sambit Bhattacharyya
Mohammad Bitar
Andrea Boltho
John P. Bonin
Valerija Botrić
Vincent Bouvatier
Josef C. Brada
Michael Brei
Gregory Brock




\begin{tabular}{|c|c|c|}
\hline Tsun Se Cheong & Damiano Fiorillo & Tomohiko Inui \\
\hline Angela Cheptea & Belton M. Fleisher & Małgorzata Iwanicz- \\
\hline Thomas Chiang & Veljko Fotak & Drozdowska \\
\hline Christina Christou & Jan Frait & Ichiro Iwasaki \\
\hline Jessica Clement & Richard Frensch & Arun Jacob \\
\hline Thomas Cooley & Zuzana Fungacova & Anshul Jain \\
\hline Tom Coupe & Nikhar Gaikwad & Esther Jeffers \\
\hline Nuno Crespo & Gabriele Galati & Morten Jerven \\
\hline Laszlo Csaba & Scott G Gehlbach & Ramona Jimborean \\
\hline Marek Dabrowski & Agnieszka Gehringer & Cristina Jude \\
\hline Tatjana Dahlhaus & Vicente German-Soto & Mohamed Kadria \\
\hline Thibault Darcillon & Aleksandr V. Gevorkyan & Thaqif Kamaruzdin \\
\hline Zsolt Darvas & Saibal Ghosh & Bonet Chikhawo \\
\hline Guillaume Daudin & Heather Gibson & Kamwana \\
\hline Jakob de Haan & Natacha Gilson & Desire Kanga \\
\hline Indra De Soysa & Blaise GNIMASSOUN & David M. Kemme \\
\hline Fabrice Defever & Christophe Godlewski & Michael Keren \\
\hline Francesco Di Comite & Stefan Goldbach & Enisse Kharroubi \\
\hline Amadou Bassirou Diallo & Manuela Goretti & Jaebeom Kim \\
\hline Sel Dibooglu & Daryna Grechyna & Emizet Kisangani \\
\hline Vidya Diwakar & Louise Grogan & Wee Chian Koh \\
\hline Francois K. Doamekpor & Steve Loris Gui-Diby & Emanuel Kohlscheen \\
\hline Istvan Dobozi & Thorvaldur Gylfason & Vladimir Kontorovich \\
\hline Kristin Doughty & Volkan Hacioglu & Marta Korczak \\
\hline Devon Douglas-Bowers & Miroljub Hadzic & Iikka Korhonen \\
\hline John Dove & Degol Hailu & Crt Kostevc \\
\hline Nadia Doytch & Peter Haiss & Mark Koyama \\
\hline Gilles Dufrenot & Nadav Halevi & Evan Kraft \\
\hline Kwamie Dunbar & Anais Hamelin & Tobias Kronenberg \\
\hline Mardi Dungey & Paul G. Hare & Ricardo Lago \\
\hline Nazif Durmaz & Oleh Havrylyshyn & Yoram Landskroner \\
\hline Balazs Egert & Boris Hofmann & Frank Lang \\
\hline Vasco Eiriz & Jens NA Holscher & Ricardo Laremont \\
\hline Michael Ellman & Tomas Holub & Gerard Lassibille \\
\hline Ozgur Ergungor & Carsten A. Holz & Laura Baselga Pascual \\
\hline Lennart Erixon & Seppo Honkapohja & Daniel le Maire \\
\hline Etienne Espagne & Barbara E. Hopkins & Peter Leeson \\
\hline Saul Estrin & Albert $\mathrm{Hu}$ & Emiliano Libman \\
\hline Abel Ebeh Ezeoha & Sonya Huffman & Maria Litvinova \\
\hline Martin Falk & Joseph Hughes & Julie Lochard \\
\hline Luisa Farinha & Christian Hutter & Steven Lugauer \\
\hline Edgar Feige & Thomas Hyclak & Annamaria Lusardi \\
\hline Jarko Fidrmuc & Roberto Iacono & Tomasz Lyziak \\
\hline Andrew Filardo & Nicolae-Bogdan Ianc & Paul Maarek \\
\hline George Filis & Mansor Ibrahim & Corrado Macchiarelli \\
\hline
\end{tabular}




\begin{tabular}{|c|c|c|}
\hline Daniel Makina & David Prychitko & Arilton Teixeira \\
\hline Svitlana Maksymenko & Thomas Pugel & George Tesar \\
\hline Adeel Malik & S. Qi & Andrey Timofeev \\
\hline Fola Malomo & Pablo Querubin & Kiril Tockov \\
\hline Phatima Mamardashvili & Roberto Ramos & Liang Tong \\
\hline Josue Mbonigaba & Magdaleno & Christoph Trebesch \\
\hline James McNulty & Kolleen J. Rask & Joanna Tyrowicz \\
\hline Jeffrey B. Miller & Thomas G. Rawski & Murat Üngör \\
\hline Raoul Minetti & Iveta Řepková & Milica Uvalic \\
\hline Kalman Mizsei & Youngwoo Rho & Neven Valev \\
\hline Alexander Muravyev & Bjorn Richter & Don Van Atta \\
\hline Ryan H. Murphy & Felix Rioja & Freddy van den Spiegel \\
\hline Peter Murrell & Martin Rode & Enrico Vanino \\
\hline Mihai Mutascu & Gerard Roland & Carole Verne \\
\hline Cephas B Naanwaab & Fabien Rondeau & Ilya Borisovich \\
\hline Usha Nair-Reichert & Barkely Rosser & Voskoboynikov \\
\hline Kanda Naknoi & Marina V. Rosser & Goran Vuksic \\
\hline Hiranya Nath & Peter L. Rousseau & Paul Wachtel \\
\hline Gisle James Natvik & Ole Rummel & Helmut Michael Wagner \\
\hline Christina Nicolas & Nahla Samargandi & Michael Wedow \\
\hline Simona Nistor & Uma Sankaran & Laurent Weill \\
\hline Guillermo Noguera & Nagham Sayour & Stephen Whelan \\
\hline Godwin Okafor & Friedrich Schneider & Wesley Widmaier \\
\hline seun Olayungbo & Dirk Schoenmaker & Gary Winslett \\
\hline Ararat L Osipian & Maria Semenova & Michael Witt \\
\hline Michael Overesch & Bruno S. Sergi & Karl Wohlmuth \\
\hline Ozde Oztekin & Unal Seven & François-Charles Wolff \\
\hline Ugo Panizza & Yochanan Shachmurove & Shane Worner \\
\hline Vasileios Pappas & Syed Shahabuddin & Tsung-Pao Wu \\
\hline Roberto Patuelli & Parmendra Sharma & Yanbing Wu \\
\hline Michal Paulus & Oleksandr Shepotylo & Yanrui $\mathrm{Wu}$ \\
\hline Carmelo Petraglia & John Simister & Lihong Yang \\
\hline Marjan Petreski & Bo Sjö & Canan Yildirim \\
\hline Yuri Pettinicchi & Helen O. Solomon & Thierry Yogo \\
\hline Russell Pittman & Bent Sorensen & Angelo Zago \\
\hline Miroslav Plasil & Martin C. Spechler & E. Zervas \\
\hline Zbigniew Polanski & Karsten Staehr & Kevin Zhang \\
\hline Richard Pomfret & Pekka Sutela & Tian Zhu \\
\hline Cristian Popa & Richard Sylla & Muhamed Zulkhibri \\
\hline Jonas Prager & David Tarr & \\
\hline Mariusz Prochniak & Sheida Teimouri & \\
\hline
\end{tabular}

Publisher's Note Springer Nature remains neutral with regard to jurisdictional claims in published maps and institutional affiliations. 